Revue internationale P.M.E.

Économie et gestion de la petite et moyenne entreprise

\title{
PME et districts industriels : quelques réflexions critiques à propos «du modèle italien»
}

\section{Bernard Ganne}

Volume 2, numéro 2-3, 1989

URI : https://id.erudit.org/iderudit/1007939ar

DOI : https://doi.org/10.7202/1007939ar

Aller au sommaire du numéro

Éditeur(s)

Presses de l'Université du Québec

ISSN

0776-5436 (imprimé)

1918-9699 (numérique)

Découvrir la revue

Citer cet article

Ganne, B. (1989). PME et districts industriels : quelques réflexions critiques à propos «du modèle italien». Revue internationale P.M.E., 2(2-3), 273-285.

https://doi.org/10.7202/1007939ar
Résumé de l'article

La mise en avant actuelle des districts industriels italiens comme nouveau modèle de référence ne risque-t-elle pas de contribuer à entretenir en fait un mythe et à occulter ce qui constitue peut- être l'originalité et la spécificité de ces systèmes ? Au-delà du " modèle» prôné et des « success stories» faites actuellement, l'important n'est-il pas aujourd'hui de mieux rendre compte de la diversité de ces ensembles, de leurs fluctuations historiques et donc de leurs dynamiques d'adaptation, aussi bien que du cadre socio-politique global qui les conditionne et dans lequel ils s'inscrivent ? Plus qu'un contre-modèle, opposé à celui de la grande entreprise, les districts italiens ne constituent-ils pas plutôt l'occasion de remettre en cause les dichotomies toutes faites (groupes/PME; local/global; économique/culturel) qui sérient et bornent peut-être actuellement les approches de l'entreprise et des systèmes industriels ?
Ce document est protégé par la loi sur le droit d'auteur. L'utilisation des services d’Érudit (y compris la reproduction) est assujettie à sa politique d'utilisation que vous pouvez consulter en ligne.

https://apropos.erudit.org/fr/usagers/politique-dutilisation/ 


\title{
PME et districts industriels : quelques réflexions critiques à propos «du modèle italien»
}

\author{
Bernard GANNE' \\ GLYSI, Université de Lyon
}

\begin{abstract}
RÉSUME
La mise en avant actuelle des districts industriels italiens comme nouveau modèle de référence ne risque-t-elle pas de contribuer à entretenir en fait un mythe et à occulter $\infty$ qui constitue peutêtre l'originalité et la spécificité de ces systèmes ? Au-delà du «modèlen prôné et des asuccess stories" faites actuellement, l'important n'est-il pas aujourd'hui de mieux rendre compte de la diversité de ces ensembles, de leurs fluctuations historiques et donc de leurs dynamiques d'adaptation, aussi bien que du cadre socio-politique global qui les conditionne et dans lequel ils s'inscrivent ? Plus qu'un contre-modèle, opposé à colui de la grande entreprise, les districts italiens ne constituent-ils pas plutótl'occasion de remettre en cause les dichotomies toutes faites (groupes/PME; local/global; économique/culturel) qui sérient et bornent peut-être actuellement les approches de l'entreprise et des systèmes industriels ?
\end{abstract}

\begin{abstract}
Presently, the Italian industrial districts are put forward as the new reference model. Does this practice run the risk of effectively fostering a myth and overshadowing precisely the elements that make up the originality and specificity of these systems ? Beyond today's much talked-of «model» and the success stories, is it not more important to understand better their diversity,
\end{abstract}

- Sociologue et chargé de recherches au CNRS, directeur pendant trois ans de la Revue «Economie et Humanisme», il est membre du GLYSI (Groupe Lyonnais de sociologie industrielle), où il a plus particulièrement centré ses recherches sur l'étude des systèmes industriels locaux. Bernard Ganne est l'auteur de plusieurs ouvrages et de nombreux articles: il prépare actuellement un film montrant les mutations récentes d'une aire d'ancienne industrialisation faisant l'économie de l'étape tayloriste.

Adresse: Groupe Lyonnais de Sociologie Industrielle, Université Turnière Lyon 11, M.R.A.S.H., 14, ave. de Berthelot 69363 Lyon cédex 7, FRANCE 
historical fluctuations and thus their process of adaptation, as well as the global socio-political framework which conditions them and within which they operate ? Rather than being a countermodel which contrasts the big firm model, do the Italian districts provide an opportunity to question the ready-made dichotomies (groups/SME; local/global; economic/cultural) which currently classify and perhaps restrict enterprise and industrial system approaches ?

\section{RESUMEN}

El enfoque puesto en los districtos industriales italianos como nuevo modelo de referencia hace correr el riesgo de mantener un mito y de esconder lo que constituye, quizàs, la originalidad y la espicifidad de estos sistemas. Màs allà del modelo alabanzado y de las "success stories", hoy, lo importante es de dar cuenta, en forma mejor, de la diversidad de las experlencias, de sus fluctuaciones històricas $y$, por ende, de sus dinàmicas de adaptaciòn dentro del marco sociopolitico global que las condiciona y en el cual se arraigna. Mucho màs que un contra-modela opuesto al de la grand empresa, los districtos italianos son una oportunidad para cuestionar las dicotomias tradicionales (grupo/PYME; local/global; economico-cultural) que fragmentan y limitan, en la actualidad, los modos de analisis de la empresa y de los sistemas industriales. 


\section{Introduction}

Quels enseignements tirer du type de développement des PME observé en Italie ? Quelle généralisation attribuer au phénomène des districts industriels ? En un mot, quelle portée reconnaître au modèle d' «industrialisation diffuse»de l'Italie du milieu? Telles sont effectivement quelques-unes des questions qui se posent au moment où l'ampleur du phénomène, sa réussite et sa permanence semblent avoir établi qu'il ne s'agit aucunement d'une phase de développement marginale ou temporaire, mais bien d'un mode de structuration spécifique à prendre en compte comme tel.

Parallèlement, on le sait, l'évidence des limites auxquelles ont mené depuis quelques années, les perspectives de l'économie concentrée et les rigidités structurelles qu'elles semblaient perpétuer dans la crise n'ont pas peu contribué à aviver l'intérêt que l'on observe aujourd'hui pour les PME et les districts industriels italiens. L'engouement actuel s'avère d'autant plus grand que la désillusion par rapport aux modèles prônés antérieurement a été forte. La «flexibilité» n'est-elle d'ailleurs pas en train de devenir le nouveau maître-mot qui s'impose? Et, l'avantage du cas italien n'est-il pas précisément de fournir un exemple fonctionnant en quelque sorte comme avant la lettre?

Mais c'est peut-être précisément ce qui fait problème. La «relecture» actuelle du cas italien et sa mise en avant comme nouveau modèle de référence pris plus ou moins hors contexte ne risque-t-elle pas d'aboutir à occulter ce qui constituait peut-être l'originalité et la spécificité du processus de développement observé là ? L'inversion actuelle de problématique à laquelle nous assistons dans la pensée économique («PME» en lieu et place des «groupes», «flexibilité des réseaux informels» face à la «rigidité des grandes structures organisées», systèmes «locaux» plutôt que «globaux» etc...) ne dévoye-t-elle pas ainsi quelque peu l'exemple italien pour l'intégrer sans autre forme de procès à la nouvelle systématique ? Quitte donc à en faire le nouveau «modèle» et quitte donc à détourner ce faisant de l'analyse des conditions concrètes de production de ce dernier.

Notre proposn'estévidemment pas ici de nier l'importance des phénomènes observés dans l'Italie du Nord-Est-Centre et le regain d'intérêt porté à partir de là aux districts industriels et aux systèmes industriels locaux (cf. en particulier Becattini, 1987). Il viserait plutôt à montrer combien un certain type d'idéalisation du phénomène tendant précisément à ériger les phénomènes observés dans la troisième Italie en «un nouveau modèle de développement» contribue en faità occulter ce qui constitue à notre sens la dynamique de ces phénomènes et à travestir du même coup les enseignements qui peuvent donc en être tirés.

C'est en tout cas ce que nous souhaiterions brièvement développer ici en montrant comment l'inversion de perspective à laquelle nous assistons maintenant, fétichisant quelque peu l'exemple italien, ne permet aucunement de transformer les perspectives économicistes qui avaient pu aboutir antérieurement à méconnaître 
purement et simplement les PME aussi bien que les systèmes locaux. Avec donc le même risque de désillusion qu'auparavant et sans plus permettre un renouvellement des approches. Or, n'est-ce pas jusqu'à une approche renouvelée des systèmes industriels et à une remise en perspective des approches économiques que semble devoir mener l'observation des processus se développant en Italie?

Le problème est que bien souvent, le phénomène des districts est considéré comme un acquis dont on part pour développer des considérations générales, plus que comme un construit, dont il convient d'analyser de façon précise les conditions concrètes de possibilité.

Dans leur soucis d'affirmer la réalité de leur objet, un certain type d'études développé en Italie a sans doute grandement contribué à accréditer l'idée qu'il s'agissait bien là d'un modèle dont on avait le soucis de montrer les conditions générales de possibilité plus que l'enracinement concret. Sans remettre en cause ici tout l'intérêt de l'abondante littérature développée autour des districts industriels, du modèle italien de PME et de la flexibilité de ce modèle, ni des progrès théoriques ainsi occasionnés, force est cependant de reconnaître que le volume des études monographiques précises ne semble pas tout à fait à la hauteur du volume de réflexions générales auxquelles celles-ci ont pu donner lieu. Et si l'on descend dans les premières jusqu'au niveau de la méthode, combien de descriptifs monographiques s'en tiennent à enregistrer de grandes concomitances générales semblant comme dispenser d'étudier plus avant les formes d'organisation concrètes des forces économico-sociales : présents sur les zones étudiées, métayage et formes d'organisation familiales se transforment bientôt en critères du «modèle» sans que l'on ait toujours bien poussé la démarche jusqu'à savoir par quels canaux concrets on allait en fait de la première structure à la seconde... De la même façon, on parle beaucoup «d'aires-systèmes» ou de districts, mais sans jamais tellement approfondir le ou les systèmes de relations qui «font systèmes» et selon quelles règles ils le font et selon quelle dynamique... N'est-ce donc pas ce modèle de lecture qu'il conviendrait maintenant de reprendre et d'approfondir?

D'autant qu'à l'extérieur, l'exportation du «modèle» n'est pas à notre sens sans entretenir confusion et ambiguïtés. N'a-t-on pas vu récemment un très dynamique colloque consacré aux «nouvelles formes d'industrialisation» brandir avec enthousiasme le modèle italien et, subjugué par la redécouverte des PME et du local, en proclamer le primat et l'universalité. A Cholet, Sfax, Chambéry aussi bien qu'à Prato ou Carpi,n'est-ce pas un même type de dynamisme local qui s'est avéré être la clé du succès et du développement?

Loin de nous aider ainsi à appréhender l'originalité des phénomènes une certaine façon de poser la question et de quêter «le-modèle-italien-de-PME» ne contribue-t-elle pas en fait à entretenir le mythe? De là, on effectuerait une série de réductions.

- Admise sans autre forme de procès, la présupposition d'un modèle unique ne contribue-t-elle pas à occulter la variété du phénomène, dans ses dimensions tant 
historiques que géographiques?

- Par ailleurs, la recherche par trop exclusive de «modèles» n'incite-t-elle pas à figer quelque peu les processus observés, privilégiant l'étude des formes sur celle des dynamiques de production et montrant donc peu, de ce fait, l'évolution relative de ces dernières?

- Enfin, la focalisation sur l'évidence du «local» et des «PME» n'impose-t-elle pas d'emblée un registre de réponse par trop exclusivement rivé sur l'endogène au détriment du politique ?

Sans entrer certes dans des débats aussi scolastiques que ceux développés, par exemple, par A. Amin et K. Robins (1989) à propos de la réalité autonome des districts, nous nous efforcerons plutôt ici de montrer quelques-unes des apories que développe, à notre sens, la quête actuelle du «modèle italien de PME» en essayant de poser quelques questions sur les limites actuelles dans lesquelles on semble enfermer ce modèle.

\section{Au-delà du modèle unique, retrouver la diversité}

Si l'on s'accorde généralement à reconnaître que le modèle de développement observé dans l'Italie du milieu n'est peut-être pas aussi unifié qu'il y paraît, et si certains chercheurs italiens, tel en particulier Bagnasco, ont toujours bien pris soin d'affirmer cette diversité, on doit reconnaître que l'ensemble des débats actuels semble souvent présupposer l'inverse : le titre même de ce dossier n'en constitue-til pas une bonne illustration?

\subsection{De la «troisième ltalie» au «modèle NEC»}

Unicité du modèle? Ce raccourci commode peut certes fort bien s'expliquer si l'on considère, par exemple, les conditions dans lesquelles la question des districts industriels a pu progressivement prendre corps en Italie : n'avait-il pas alors fallu batailler vigoureusement pour faire admettre que les phénomènes observés dans l'Italie du Centre ne relevaient pas d'une seule période de transition ou d'un simple avatar de la décentralisation mais bien d'un phénomène de développement spécifique à prendre en compte comme tel (Becattini, 1987) ? D'où l'affirmation de la consistance du phénomène, de son extension géographique (les travaux de Sforzi, 1988), de sa cohérence économique (Brusco 1982, 1986; Garofoli, 1981), de sa validité comme modèle de référence plus large (Fua, 1983) etc.; et les «districts» de se métamorphoser en «modèle NEC» (pour Nord-Est-Centre de l'Italie : Fuà, 1985).

Quelles que soient ainsi les réserves admises verbalement, c'est donc jusqu'ici plus sur la généralité du phénomène que sur sa diversité que semble s'être polarisée l'attention. 


\subsection{L'unité commode...}

L'affirmation pugnace de la réalité du modèle n'a-t-elle pas amené à en survaloriser quelque peu l'unité ? D'autant que, reprise hors de la Péninsule, cette présentation n'est pas sans s'être trouvée encore quelque peu durcie.

Les districts italiens ne sont-ils pas alors apparus à l'extérieur de l'Italie comme «le» modèle de référence permettant aux chercheurs d'échapper aux impasses des paradigmes économiques dominants de la concentration tout en fournissant aux praticiens une sorte de vivant exemple de réponse à la crise : double accréditation incitant plus, là encore, à se polariser sur le monolithisme de ce modèle NEC qu'à porter attention à la diversité du phénomène.

Tant dans les articles scientifiques que dans les revues opérationnelles, le modèle italien de PME n'a-t-il pas souventété présenté comme cette sorte de référent unique paré de toutes les vertus d'efficacité, de cohésion sociale et d'adaptation flexible, tous lieux et temps presque confondus? Avec ses modèles-phares, tels que Prato ou Carpi, censés en quelque sorte en concentrer les vertus...

\section{$2.3 \quad$...d'un phénomène multiforme}

Or, ne serait-il pas temps maintenant de poursuivre le travail et d'arrêter l'amalgame? Ne conviendrait-il pas de réfléchir plus avant sur la parenté ainsi préétablie entre des situations aussi diverses que celles observées dans des zones d'ancienne structuration industrielle comme Prato et d'autres zones peut-être voisines de Toscane ne connaissant un décollage industriel que dans la période récente du post après-guerre ? L'intérêt de ces autres zones - et une des clés de leur succès n'est-il pas précisément de ne pas avoir eu de tradition industrielle ? Et la mise en avant de ce que l'on a pu appeler «l'exaspération de la diffusion industrielle» du modèle Prato et de ses «impannatori» (Ritaine, 1987) ne risque-t-elle pas alors de dissimuler l'originalité propre de ces derniers? Le problème peut sans aucun doute être également étendu aux autres districts et régions.

Alors que s'avère désormais reconnue l'originalité du modèle de développement observée dans l'Italie du milieu, n'aurait-on pas aujourd'hui intérêt à bien différencier les diverses formes prises par le phénomène, à bien en isoler les divers niveaux ? Et n'est-ce pas précisément la question à poser aujourd'hui ?

\subsection{L'exemple Italien : modèle ou anti-modèle ?}

S'il ne renvoyait qu'à une lecture un peu rapide du phénomène, l'amalgame évoqué serait au fond sans importance. Le problème est que l'idéalisation active du modèle italien à laquelle nous assistons développe, à notre sens, une lecture à contresens de l'expérience italienne. 
La mise en avant par trop inconditionnelle du «modèle» n'est-elle pas en fait ce qui empêche de percevoir que l'un des intérêts de l'exemple italien réside moins dans «le» modèle qu'il représente que dans la variété des systèmes qu'il a permis de laisser se développer et dans la diversité des réponses ainsi apportées aux problèmes qui se posaient? Plus que dans un modèle, une part de la «flexibilité» dont il est tant parlé à propos de l'Italie ne tient-elle pas précisément à cette variété qui a su se développer et subsister, et pas seulement d'ailleurs au niveau des PME ? N'est-ce pas cette flexibilité des réponses et ces formes de construction sociale différenciées du local dans l'espace comme dans le temps qu'il conviendrait d'analyser au-delà de la quête concernant la structure fournissant la clé de l'organisation de ces divers districts?

\section{Au-delà des "success stories" des districts, retrouver les dynamiques historiques}

Rendre mieux compte de la variété des districts; donc, rendre aussi mieux compte de leurs dynamiques et de leurs évolutions. Ne touchons-nous pas là à un deuxième type d'impasse affectant la présentation actuelle des districts industriels italiens?

\subsection{Les districts ou la voie du succès}

N'est-ce pas en effet surtout une «success story» qui nous est pour l'instant délivrée là ? Valorisant certes tout le contre-exemple que les districts peuvent représenter comme modes d' organisation locaux plus ou moins formels faisant pièce aux normes économiques précédemment admises mais n'abordant qu'assez peu les blocages provoqués, les difficultés rencontrées ou les aspects négatifs, bref, semblant comme ignorer l'envers du décor, «disparitions» de districts, «déclins» de districts, «crises» ne semblent pas avoir leur place dans un modèle donné souvent comme naturellement triomphant...

S'il est admis que les districts n'ont pas échappé à la crise, s'il est reconnu ici ou là que certains ont pu plus ou moins sombrer ou être supplantés, si même quelques doutes peuvent percer quant à leur avenir, force est de reconnaître que peu d'études ont contribué à opérer une présentation dynamique - et donc contrastée de l'histoire de ces districts.

Les systèmes étudiés semblent ainsi donnés d'emblée comme efficients; et c'est d'ailleurs moins leur mode de structuration propre - avec ses avatars, ses revers, ses retournements - qui se trouve étudié que les conditions de possibilité globales de leur efficience qui sont de fait habituellement analysées. Les «aires- 
systèmes» ne semblant pas faire de doute, c'est moins l'analyse concrète de leurs modes propres d'organisation et de relations qui fera l'objet d'analyses précises (ce qui ne s'avérerait pas inutile pour savoir en quoi précisément elles font systèmes) que l'étude des conditions générales permissives leur ayant donné lieu (conditions de marché, d'environnement etc....). On continue ainsi toujours d'ignorer les systèmes de relations que les entreprises peuvent développer entre elles, ainsi que les systèmes de régulation de ces relations et de celles qu'elles entretiennent précisément avec les autres instances ou institutions sociales ou politiques locales. On sait tout de la flexibilité d'ensemble de ces systèmes, mais, en l'état actuel des travaux, eux-mêmes ne restent-ils pas pour partie une coque vide?

La mobilisation réalisée par les districts apparaît ainsi comme une sorte de donnée de base, un présupposé premier dont on tentera d'étudier les conditions d'ensemble (culturelles, économiques ou familiales) occultant les blocages, voire ce que l'on pourrait appeler les phénomènes de déconstruction du local. Face à cette prégnance et à cette évidence du modèle, tout phénomène contraire ne risque-t-il pas d'apparaître comme un simple accident à la marge, comme ce fut le cas il n'y pas si longtemps avec la «loi» de la concentration?

\subsection{Construction, déconstruction...}

Si l'étude des districts s'est ainsi révélée prolixe dans l'analyse de ce que l'on pourrait appeler les «conditions de construction du local», elle s'avère peu bavarde sur ses conditions de déconstructions, voire sur les simples évolutions. Or, retournant quelques années après en Toscane avec $\mathrm{A}$. Michelsons et $\mathrm{F}$. Baptiste (1988), nous avons vu comment l'intérêt d'une zone comme celle du district de la Val d'Elsa était précisément d'avoir connu des phases d'évolution fortement différenciées par rapport à ce qui avait pu être observé il y a quelques années par Bagnasco et Trigilia (1984), montrant comment les phénomènes de «diffusion industrielle», loin de permettre d'échapper à la crise, avaient pu accentuer les effets de cette dernière de par la rupture du consensus social qui avait pu alors s'opérer en accélérant les écarts sociaux. Mais c'est aussi cet impact accentué de la crise qui permettrait peut-être d'impulser actuellement - et de hâter peut-être - un nouveau mode d'organisation et de concertation plus formalisé sur une base territoriale locale élargie. L'histoire de la Val d'Elsa, c'est aussi l'histoire des crises traversant ce secteur de Toscane et des crises de l'organisation socio-territoriale faisant et défaisant successivement la zone. Bagnasco comme Trigilia, pour leur part, n'avaient pas été sans s'interroger sur la fragilité et la relativité des conditions qui, un temps, avaient permis à cette région de connaître une importante industrialisation à base de PME. Il est d'autant plus regrettable que, de ces analyses résolument contextuelles, n'ait le plus souvent été conservé qu'un «modèle», une sorte de système idéal d'optimisation des ressources tant humaines qu'économiques qu'il ne resterait plus qu'à adapter... 


\subsection{Des modes de réponses différenciées}

Ne sommes-nous pas floués ici par la façon d'axer la question sur la recherche de structures et de modèles plutôt que sur l'analyse de processus ? La recherche d'un «modèle» n' incite-t-elle pas d'emblée à se situer dans une perspective qui s'abstrait de l'histoire, à la recherche de structures d'organisation pouvant s'appliquer généralement et comme de façon intemporelle (à l'image des normes économiques «universelles» ? Pourtant, l'efficacité des districts industriels italiens réside dans le type de réponse spécifique apportée par un mode d'organisation économique et social dans des contextes et une conjoncture bien précises, lesquels semblent s'être considérablement transformés au cours du temps. La diffusion industrielle observée après-guerre dans les districts n'a ainsi que peu à voir avec les phénomènes de re-territorialisation observés dans la période récente suite à la crise, ceci sans même parler des types de développement actuels à base de flexibilité. Mais ne se heurte-t-on pas là au manque de monographies précises montrant les dynamiques concrètes d'articulation des acteurs sociaux et des institutions locales et se cantonnant à observer comme il a déjà été dit de grandes concomitances d'ensemble.

Ne convient-il pas dès lors de déplacer la problématique de la quête de structures plus ou moins «modèles» vers celle des processus de réponse historiquement observés au travers de l'évolution des districts qui naissent, meurent, peuvent disparaître, alterner, etc. N'est-ce pas ce nouveau champ d'analyse qui permettrait d'échapper au cercle tautologique des «success stories» concernant les districts industriels, en montrant tout l'intérêt en même temps que les limites (ou au travers même des limites...)? Plus que par le modèle idéal qu'ils représentent, Prato et Carpi ne nous instruisent-ils pas mieux par les problèmes auxquels ils se heurtent actuellement et par la façon dont ils s'efforcent - avec ou sans succès - de les résoudre ? Plus que leur succès, n'est-ce pas leur vulnérabilité et la conscience qu'ils peuvent avoir de cette dernière qui peut rendre sensible le type de construction original et spécifique qu'ils présentent?

Nécessité donc, au-delà des grands paradigmes généraux habituellement invoqués pour rendre compte des districts (rôle du métayage, de la famille, de l'organisation sociale et culturelle...) de réinsérer ces derniers dans des perspectives historiques plus dynamiques et moins infaillibles, permettant d'échapper à la fétichisation s'attachant actuellement aux systèmes locaux. Ce qui implique certes un autre type d'approche des phénomènes «locaux» et de leur construction, de par un autre type de mise en perspective de l'économique et du social. 


\section{$4 \quad$ Au-delà des dichotomies toutes faites, recomposer les approches}

La façon de privilégier actuellement au travers des «districts industriels italiens» aussi bien les formes d'organisation locales que les PME, ne renvoie-t-elle davantage à la mauvaise conscience d'avoir trop ignoré ces domaines dans le passé qu'à un soucis d'échapper aux perspectives qui avaient permis d'aboutir à de telles impasses?

Selon nous, le problème n'est pas de remettre au centre, en lieu et place des précédentes, ces catégories oubliées, mais bien de transformer le cadre d'analyse qui avait contribué à les ignorer.

La question n'est pas tant de remplacer dynamiques des groupes par dynamique des PME, structurations globales par structurations locales, déterminations économiques par déterminations culturelles, mais d'opérer une révision des dichotomies inhérentes à ce type de catégories afin de tenter de trouver au-delà d'elles, ce que leur découpage séparait.

\subsection{Groupes contre PME ou analyse des formes différenciées d'organisation Industrielle?}

Au lieu d'exaspérer l'opposition groupes/PME, le déplacement de problématique à opérer aujourd'hui ne réside-t-il pas plutôt dans la mise sur pied d'un système d'approche de la variété des formes d'organisation industrielles que peuvent se donner les entreprises, groupes comme PME ? Plus que ce vague critère de taille "grand/petit» n'est-ce pas finalement la variété de ces formes jouant tant au niveau interne que dans les systèmes de relations mis en place entre les entreprises et déterminant de ce fait de nouveaux types «d'unités» qu'il s'avère pertinent d'analyser ? Il existe des PME tout à fait fordistes, bâties sur le type de modèle intégrateur de la grande entreprise, qui n'apprennent en fait pas grand chose de neuf sur les nouvelles formes d'organisation industrielles, de même qu'il existe divers types de groupes, qui, quelles que soient pourtant les contraintes communes subies par le marché, ne se construisent pas selon le même type de modèle. Des études récentes, effectuées précisément entre autres en Italie, ne viennent-elles pas de montrer combien, dans la même région, des groupes comme Fiat ou Olivetti pouvaient développer des modèles d'organisation totalement contrastés et des politiques locales opposées (Michelsons, 1989) ? Le choix fordiste de Fiat n'apparait ainsi que comme l'un des choix politiques possibles dans une panoplie où le système Olivetti semble avoir pour sa part privilégié un tout autre type de dynamique. Et les réflexions actuelles autour de la flexibilité ne sont-elles pas en train de montrer que, plus qu'un problème d'opposition entre les groupes et les PME, c'est peut-être bien de nouveaux systèmes de relations induisant d'autres types de configurations 
industrielles et de régulations qui constituent aujourd'hui le phénomène important caractéristique des mutations en cours. Au-delà des PME, n'est-ce pas ainsi la variété des configurations industrielles et de leur systèmes de relations et de régulations, aussi bien inter-entreprises que dans leur rapport à l'environnement local, que l'exemple italien nous invite à penser ?

\section{2 «Local» contre aglobal» ou étude des formes de construction politique du «local»?}

Au niveau de l'opposition global/local, le problème nous semble moins de percevoir en quoi une structuration «locale» particulière peut bien s'opposer à l'inéluctabilité des déterminations «globales» que de découvrir comment en fait, un certain type de système politique construit en fait simultanément le «local» et le «global».... Les deux allant précisément de pair et faisant système et le système «notable» français, tel que décrit par J.P. Worms (1966) comme caractéristique ici des échelons locaux, s'avérant indissociable de la structure globale développée par l'État centralisé français, le système des districts observé en Italie ne s'explique pas, pour sa part, sans référence à ce que l'on pourrait appeler «l'a-centralisation» de l'État italien. Plus donc qu'un en-soi à absolutiser comme tel et comportant comme en lui-même ses propres règles, l'important, relativement dissimulé aujourd'hui, n'est-il pas de voir en quoi dans chaque pays le «local» est bien un «construit» de chacun des systèmes?

\section{3 «Culturel contre économique», ou formes de construction sociale de l'économie?}

En ce qui concerne enfin l'économique opposé au social ou au culturel, le problème n'est-il pas, actuellement, au lieu de reconduire les impasses de ce type d'opposition, de comprendre les formes sociales de construction de l'économique aussi bien que le système d'échanges légitimes de biens pouvant certes également être économiques, que constitue le système social d'une société ? Formes de construction sociale des marchés, des systèmes de concurrence aussi bien que d'échange, n'est-ce pas effectivement cette sociologie économique qu'amorce toute une partie de la réflexion sur l'exemple italien (Bagnasco, 1988) mais que continue d'occulter la mise en avant de modèles?

En d'autres termes, la dynamique sociale de changement n'est-elle pas précisément dans la transgression du type de catégories ainsi sédimentées à un moment donné ? Et le travail du sociologue n'est-il pas précisément de suivre ces transgressions où se refond la société ?

Quoi qu'il en soit est-ce que les enseignements à tirer ainsi des processus observés en Italie ne sont pas à trouver : plutôt que dans les PME, dans les formes 
variées d'organisation tant internes qu' inter-entreprises observées dans les districts; plutôt que dans les modèles «locaux», dans le système d'autonomie dispensé par le modèle politique italien; plutôt que dans les particularités culturelles, dans les formes variées de construction de l'économique et leur intégration au système d'échange social légitime où elles se trouvent incluses...

Ne retenir dans l'exemple italien que les PME, les districts ou les particularités culturelles, n'est-ce pas prendre en fait la forme pour le fond et se priver du même coup de la capacité de comprendre que la spécificité du système consiste précisément dans la faculté de se construire comme un tout viable sans en référer d'abord à un système de régulation univoque?

N'est-ce pas en fait toute l'ambiguïté de la fascination exercée par l'exemple italien par rapport à l'hexagone qui amène à chercher dans la Péninsule le secret de nouveaux moyens d'intervention et de régulations alors même que la flexibilité des réponses observées en Italie réside peut-être précisément dans le fait de ne pas être régulé par un système politico-économique centralisé ?....

\section{BIBLIOGRAPHIE}

Amin, A. et Robins, K., (1989), Industrial districts and regional development : limits and possibilities, Centre for Urban and regional Development Studies - University of Newcastle upon Tyne, polycopié, 57 p. février.

Antonelli, C., Cappellin, R., Garofoli, G., et Jannaccone Pazzi, R., (1988), Le politiche di sviluppo locale, Franco Angeli, Milano, 292 p.

Bagnasco, A., (1977), The Italie : la problematica territoriale dello sviluppo italiano, Bologne, II Mulino, $255 \mathrm{p}$.

Bagnasco, A., (1988) La construzione sociale del mercato : studi sullo sviluppo di piccola impresa in Italia, Bogogne : Ed. II Mulino, 194 p. (Studi e ricerche : CCXL)

Bagnasco, A. et Trigilia, C., (1988), Società e politica nell aree di piccola impresa : il caso della Valdelsa, Milan, Franco Angeli.

Bagnasco, A. et Trigilia, C., (1984), Società e politica nelle aree di piccola impresa : il caso di Bassano, I.R.E.S., Fondazione Corrazin, Ed. Arsenale.

Becattini, G. (éd.),(1987), Mercato e forze locali : il distretto industriale, Bologne, II Mulino, 193 p. (Quaderni de «L'industria»).

Brusco, S., (1982), «The Emilian Model : productive decentralisation and social integration», Cambridge Journal of economics, no 6.

Brusco, S., (1986), «Small firms and industrial districts : the experience of Italy in New firms and regional development» dans Europe, D. Keeble et Z. Weyer (éd.), Londre, Croom Helm.

Capecchi, V.,(1987), «Les transformations du petit entreprenariat dans une région de l'Italie : parcours masculins et féminins", Annales de Vaudresson 1, no 26, pp. 243-258.

Capecchi, V., (1988a), «Les facteurs de développement d'une économie régionale», polycopié, $43 \mathrm{p}$. 
Capecchi, V., (1988b), «La mobilita sociale in Emilia Romagna», II Mulino, Bologne, 428 p. Fuà, G.,(1985), «Les voies diverses du développement en Europe», Annales Économies Sociétés Civilisations, no 3, mai-juin, pp. 579-603.

Fuà, G. et Zacchia, C., (éd.), (1983), Industrializzazione senza fratture, Bologne, II Mulino, $334 \mathrm{p}$.

Ganne, B., (1985), «Du notable au local : transformations d'un modèle politique», Les annales de la recherche urbaine, no 28, octobre, pp. 23-32.

Ganne, B.,(1987), «Pour une sociologie des PME ou de l'entreprise comme articulation de systèmes de relations : de quelques relations préalables», Technologies, Idéologies, Pratiques, vol. VI, no 4 et vol. VII, no 1, pp. 129-147.

Ganne, B., (1989), «Les relations industrielles et leur contexte : à propos du politique comme «contexte»», Communication au colloque «Les systèmes de relations professionnelles : trente ans après J.T. Dunlop», Paris, Greco 41 «Relations professionnelles» 2-3 mars, 9 p.

Ganne, B., Saglio, J., Baptiste, F., Michelson, A. et Mac Arthur, R., (1988), Milieux industriels et systèmes industriels locaux : une comparaison France-Italie, Lyon : Groupe lyonnais de sociologie industrielle, document no 1, janvier, 194 p., annexes (Rapport).

Garofoli, G., (1981), «Lo sviluppo delle «aree periferiche» nell'economia italiana degli anni settanta», Industrial, no 11, pp. 391-404.

Garofoli, G., (1985), «Industrialisation diffuse en petite entreprise : le modèle italien des années 70», Cahiers I.R.E.P. développement, no 9, pp. 245-256.

Maruani, M., Reynaud, E., et Romani, CI., (1989), La flexibilité en Italie, Paris, Syros-Alternatives.

Maglione, R., Michelsons, A. et Rossi, S., (1989), «Autostrada numero cinque : uomini, tecnologie et imprese fra Ivrea e Torino», polycopié, $351 \mathrm{p}$.

Ritaine, E., (1985), «Ressources sociales du développement économique : un système industriel localisé, en Italie», Talence : Université de Bordeaux I, Centre d'étude et de recherche sur la vie locale (C.E.R.V.L.), 150 p. (Rapport).

Ritaine, E., (1987), «Prato ou l'exaspération de la diffusion industrielle», Sociologie $d u$ travail, no 2, pp. 139-156.

Sforzi, F., (1988), «The geography of industrial districts in Italie», Document de travail distribué lors de la rencontre du groupe de travail «Districts industriels et systèmes de coopération inter-entreprises» du B.I.T. (Genève), les 11-12 juillet 1988, 13 p.

Worms, J.P., (1966), «Le préfet et ses notables», Sociologie du travail, no 3. 